Europhys. Lett., 5 (4), pp. 327-331 (1988)

\title{
Frustration in Modulated Phases: Ripples and Boojums.
}

\author{
J. M. Carlson, S. A. Langer and J. P. Sethna \\ Laboratory of Atomic and Solid State Physics \\ Cornell University - Ithaca, N.Y. 14853, USA
}

(received 29 September 1987; accepted 30 November 1987)

PACS. 61.30 - Liquid crystals.

PACS. 64.70M - Transitions in liquid crystals.

\begin{abstract}
Many exotic modulated phases can be described in terms of regular array of defects. We have studied frustrated continuum elastic theories for several such liquid crystalline systems. While in each case the nature of the modulation and the defects is quite different, we note striking similarities in the structure of the free energies. In each case, frustration arises from the local packing properties of the molecules, and is linked to total divergence terms in the free energy. These terms can be integrated exactly to produce surface terms, which produce negative contributions to the free energy at the defects. In each case, the modulated phase is preferred when the net defect energy is sufficiently negative.
\end{abstract}

We present several exotic modulated phases which share a common feature-each has a stable phase (or phases) which possess regular arrays of defects (fig. 1). Certain lipid bilayer systems exhibit a ripple phase [1], which can be described in terms of a one-dimensional periodic density modulation composed of high-density pinched regions separated by gaps. Chiral smectic liquid-crystal films form two-dimensional patterns composed of boojums (so called because of their similarity to the boojums of superfluid ${ }^{3} \mathrm{He}-\mathrm{A}$ ) [2]. The metallic glasses [3, 4] and the blue phases of cholesteric fluids [4] have been described as networks of disclination lines.

In this letter we discuss the similarities between the continuum elastic theories for each of these phases. These theories are frustrated because the locally energetically ideal configuration of molecules cannot be extended to fill all space (fig. 2$)\left({ }^{1}\right)$. Locally, the lipid bilayers display spontaneous splay in one dimension; the smectic film displays spontaneous bend in two dimensions; and the blue phases display spontaneous twist in three dimensions. In each case, strain builds up when the ideal structure is extended. As we will show, defects (places where the molecular orientation shifts abruptly) can act to relieve this strain (fig. 3). Typically, in a physical system the defects will spread out over a few intermolecular spacings; however, the size of the defect is small compared to the length scale on which the molecular orientation varies. For this reason, most of the essential features are captured in our models which describe the limit in which the size of the defect is equal to the intermolecular spacing.

$\left.{ }^{1}\right)$ For diagrams of the metallic glasses and blue phases, see ref. [4]. 

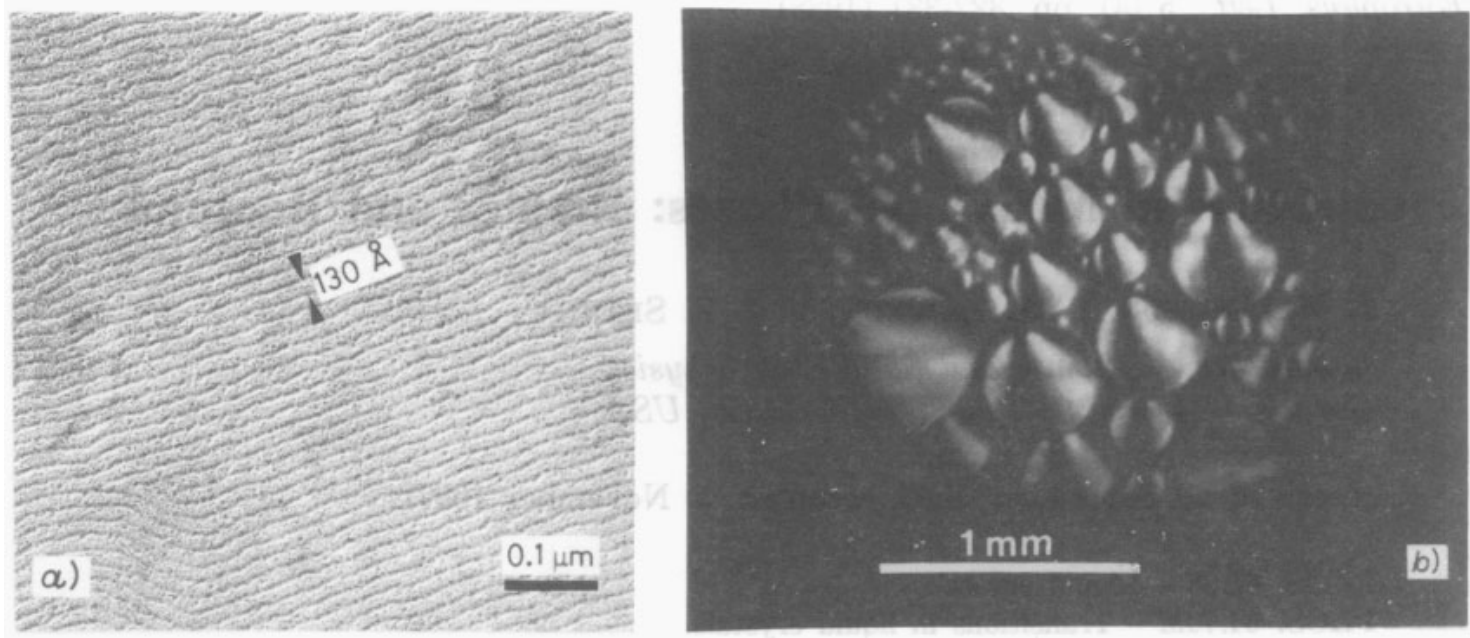

Fig. 1. - a) Freeze-fracture micrograph of the ripple phase of DPPC showing the top view of the corrugated bilayer. A cross-section perpendicular to the stripes produces a sawtoothlike pattern. (Courtesy of J. Zasadzinski and M. Schneider.) b) Micrograph through crossed polarizers of the boojums in HOBACPC. The film looks dark whenever the molecular axis is either vertical or horizontal. (Courtesy of N. A. Clark, D. H. Van Winkle and C. Muzny.)

Our first example is the smectic ripple phase $P_{\beta^{\prime}}$ which is observed in hydrated phospholipid bilayers [5]. Each molecule consists of a hydrophilic polar head group, and a pair of hydrophobic hydrocarbon chains. For certain ranges of temperature and concentration the amphiphilic nature of these molecules causes them to form bilayers. In the ripple phase the layers are corrugated, so there is a one-dimensional periodic density modulation of the chains. Figure $1 a$ ) is a typical freeze-fracture electron micrograph of the $P_{3^{\prime}}$ phase of dipalmitoylphosphatidylcholine (DPPC) [6]. The ripples have a sawtoothlike shape and the wavelength is $\sim 130 \AA$, corresponding to approximately 15 molecules [7]. The system is frustrated because the cross-sectional area of the head groups is greater than that of the associated chain pair, making it impossible to fill space without introducing some strain into the bilayer configuration, or the molecule itself.

a)
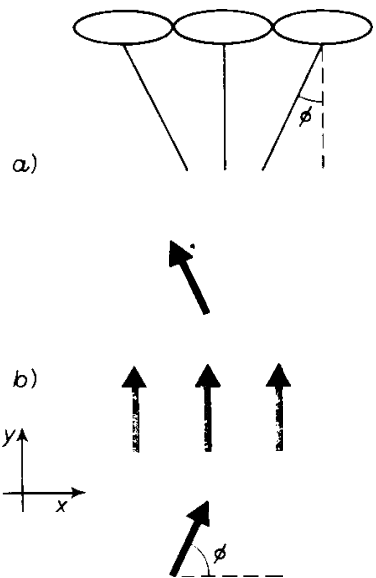

Fig. 2. - Local ideal configurations. a) Schematic DPPC molecules showing spontaneous splay of the tails. b) Projections $\hat{\boldsymbol{c}}$ of the optical axis of the HOBACPC molecules into the plane of the film, showing an inclination to bend. 

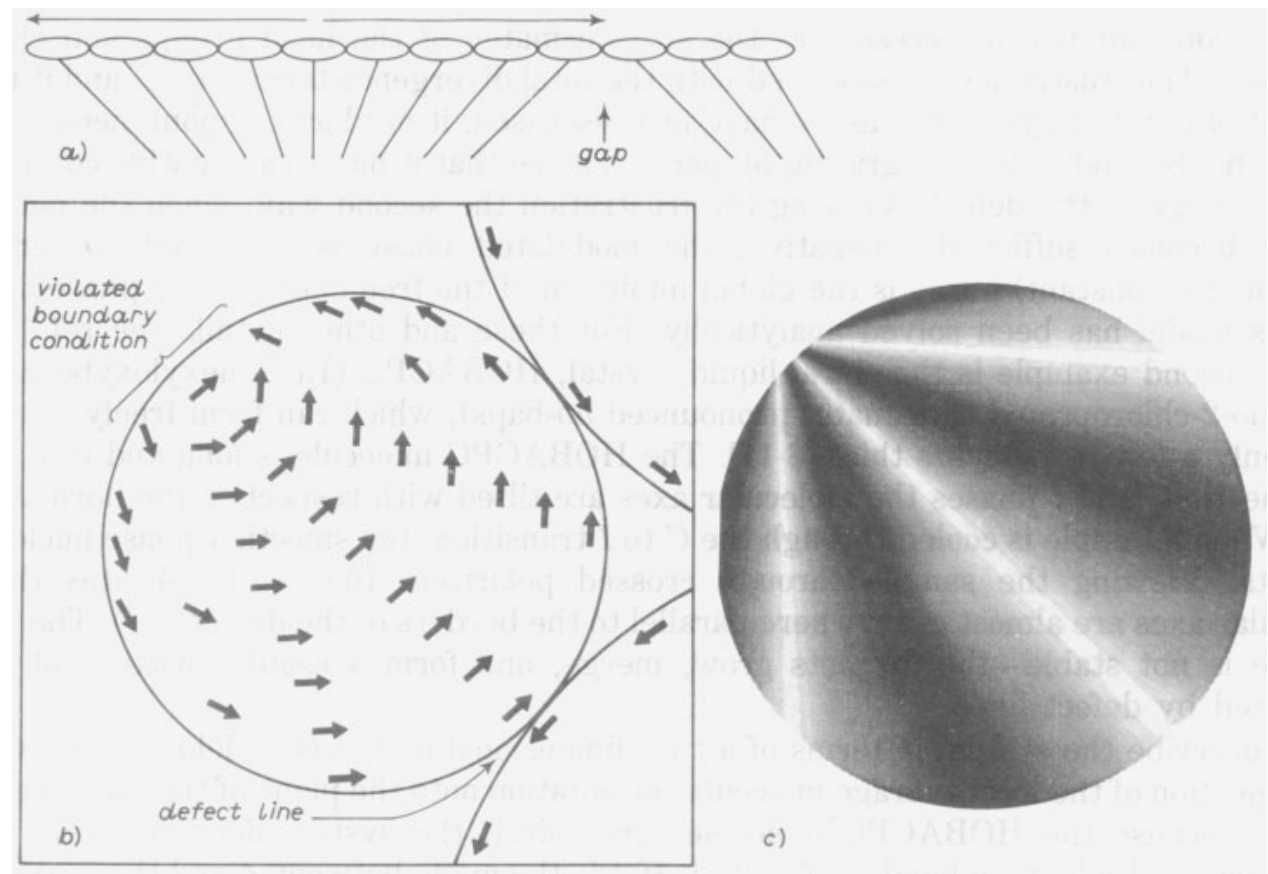

Fig. 3. - Defect configurations. a) A ripple of length $\lambda$. Bulk regions of closely packed chains are separated by gaps. $b$ ) A boojum. $\hat{c}$ is nearly parallel to the boundary almost everywhere, and there are no point defects anywhere. $c$ ) Simulated view through crossed polarizers of the boojum in $b$ ).

In our one-dimensional model [1] of the ripples the scalar order parameter $\phi$ represents the tilt of the chains with respect to the normal to the head group layer (see fig. $2 a$ )), which, for simplicity, we will assume is rigid and flat $\left({ }^{2}\right)$. As shown in table I, the free energy of this model system is a frustrated $\phi[4]$ theory with a term which represents the core energy of the defects. Because the heads are larger than the chains, adjacent molecules will have minimum energy when they tilt towards one another, giving rise to a positive gradient in the order parameter. In the modulated phase this results in regions of spontaneous inward splay of the chains, as illustrated in fig. $3 a$ ). The bulk regions are of finite length $\lambda$, and are separated by gaps (i.e. defects) which relieve the strain which builds up as $\phi$ becomes large. In the free energy, the local low-energy configuration satisfies $\partial \phi \approx q$, where $q$ is a strictly

TABLE I.

\begin{tabular}{|c|c|c|c|c|}
\hline & $P_{3}$, Phases & Chiral films & Blue phases & Metallic glasses \\
\hline $\begin{array}{l}\text { Frustrated } \\
\text { covariant } \\
\text { derivative }\end{array}$ & $\partial \dot{\phi}-q$ & $\begin{array}{l}\partial_{i f}-q c_{i} \\
(i=1,2)\end{array}$ & $\begin{array}{l}\partial_{i} n_{i}-q \varepsilon_{i j k} n_{k} \\
(i, j, k=1-3)\end{array}$ & $\begin{array}{l}\partial_{i} \Sigma_{u, z} Y_{y \nu}^{-1}-q J_{i \mu \nu} \\
(i=1-3 ; \mu, v,==1-4)\end{array}$ \\
\hline Free energy & $(\mathrm{D} \xi)^{2}+m \dot{\zeta}^{2}+\phi^{4}$ & $K_{s}(\mathrm{D} \cdot \hat{\boldsymbol{c}})^{2}+K_{b}(\mathrm{D} \times \hat{\boldsymbol{c}})^{2}$ & $(\mathrm{D} n)^{2}$ & $(\mathrm{D} Y)^{2}$ \\
\hline Total divergence & $q \partial \dagger$ & $q \partial \times \hat{\boldsymbol{c}}$ & $q \partial \cdot(\boldsymbol{n} \cdot \partial n-n \partial \cdot \boldsymbol{n})$ & $q \partial_{j}\left(\left[\Sigma \partial_{k} \Sigma^{-1}\right]_{k j}-\left[\Sigma \partial_{k} \Sigma^{-1}\right]_{j k}\right)$ \\
\hline Defects & Gaps & Boojums & $\begin{array}{l}s=-1 / 2 \\
\text { Defect lines }\end{array}$ & $\begin{array}{l}\text { Sixfold } \\
\text { coordinated } \\
\text { edges }\end{array}$ \\
\hline
\end{tabular}

$\left(^{2}\right)$ In this model, the rippling is associated only with modulation of the order parameter $\phi$. We believe that out-of-plane rippling is driven by the modulation of the chain angle when the rigidity of the layers is somewhat relaxed. 
positive constant which increases as the size mismatch of the head groups and chains is increased. The frustration is associated with the total divergence term $-q \partial \phi$, and it may be thought of in two ways. First, as we have just discussed, it produces a spontaneous splay of the chains. Second, after integrating by parts, we see that it makes a negative contribution to the energy at the defect. Viewing the frustration the second way, when the net defect energy becomes sufficiently negative, the modulated phase is preferred. Otherwise a uniform $(\phi=$ constant) phase is the global minimum of the free energy. The phase diagram for this model has been solved analytically. For these and other details see ref. [1].

Our second example is the chiral liquid crystal, HOBACPC $(\mathrm{R}(-)$ hexyloxybenzylidene $p$ '-amino-2-chloropropyl cinnamate, pronounced ho-baps), which can form freely suspended films only a few monolayers thick [8-11]. The HOBACPC molecule is long and thin, and in the smectic $C$ and $I$ phases the molecular axes are tilted with respect to the normal to the film. When a sample is cooled through the $C$ to $I$ transition, the smectic $I$ phase nucleates in droplets. Viewing the sample through crossed polarizers (fig. 1b)) indicates that the molecular axes are almost everywhere parallel to the borders of the droplets $\left({ }^{3}\right)$. The droplet texture is not stable-the droplets grow, merge, and form a regular pattern of stripes separated by defect lines.

We describe the system in terms of a two-dimensional unit vector field $\hat{\boldsymbol{c}}(x, y)$, which is the projection of the local average molecular orientation onto the plane of the film (the $(x, y)$ plane). Because the HOBACPC molecules are chiral, the system does not have mirror symmetry, and $\hat{\boldsymbol{c}}$ likes to bend $\left(^{4}\right)$ (fig. $2 b$ )). If $\phi$ is the angle between $\hat{\boldsymbol{c}}$ and the $x$-axis, then the local ideal configuration is given by $\partial \hat{\phi}=q \hat{\boldsymbol{c}}$, or, equivalently, by $\partial \times \hat{\boldsymbol{c}}=q$, where $q$ is a measure of the chirality of the system. The free energy is given in table $\mathrm{I}$.

Like the rippled phase of the lipid bilayer, the HOBACPC film is frustrated, and the frustration may be viewed in two ways. First, a unit vector field in two dimensions cannot have constant curl, so the ideal local configuration of molecules cannot be extended to cover the whole film. Second, when the total divergence term $-2 q \partial \times \hat{c}$ in the free energy is integrated by parts, it becomes the surface integral $-2 q \oint \hat{\boldsymbol{c}} \cdot \mathrm{d} l$. This term makes its maximum negative contribution to the energy when the order parameter lies parallel to the boundary of the droplet and points counterclockwise (assuming $q>0$ ). The condition for a local minimum in the free energy density, therefore, becomes a boundary condition for the droplets. If the boundary condition were to be satisfied everywhere, then there would have to be a point defect within or on the edge of the droplet. Since the core energy of such a defect is large, the defect is expelled from the droplet, violating the boundary condition slightly in the process, and producing a boojum. The boundaries of the droplets are now defect lines, because $\hat{c}$ changes orientation by $\sim 180^{\circ}$ in moving from one droplet to another, and it is these defect lines which relieve the strain of frustration. The defect lines are also the dominant feature of the stable stripe texture, which, apart from the high-order terms in the free energy, is driven by phenomena similar to the ripples in DPPC.

Two other systems in which frustration leads to defects via total divergences in the free energy are the cholesteric blue phases and the metallic glasses. Details of these systems are given in ref. [4]. In the blue phases the order parameter is a headless unit vector $n(x, y, z)$, representing the average local molecular orientation. In the locally ideal configuration $n$ has a nonzero twist, because molecules placed end to end prefer to be parallel, but molecules placed side to side prefer to have a small angle between them. The resulting defects are

${ }^{(3)}$ They could also be perpendicular, but this is probably not the case. See ref. [2]. It also does not matter.

$\left({ }^{4}\right)$ We do not know the microscopic mechanism involved here. All we can say is that the effect is not forbidden by symmetry. 
$s=-\frac{1}{2}$ disclination lines. In the locally ideal configuration of metallic glasses all atoms lie on the vertices of perfect tetrahedra. Tetrahedra cannot fill space in 3 dimensions, but they can pack on the surface of a sphere in 4 dimensions. The order parameter for the metallic glasses is the $4 \times 4$ rotation matrix $\Sigma$ that maps the local configuration of atoms into the ideal 4dimensional template. The defects in the metallic glasses are $-72^{\circ}$ disclination lines where six, rather than five, tetrahedra meet along an edge.

Our goal is a general theory of frustrated systems in the continuum limit. Table I outlines the key features we have identified so far. In each of these systems the microscopic properties of constituent molecules prescribe a locally ideal configuration which cannot fill space. This local minimum energy configuration is expressed as a preferred derivative of the order parameter. The covariant derivative $\mathrm{D} \Psi$ of the order parameter $\Psi$ is the difference between the actual derivative and the preferred derivative (see the table for specific forms) $\left(^{5}\right)$, and is a measure of how close the system comes to achieving its ideal configuration $\left({ }^{6}\right)$. In each case, the free energy density contains a term which is simply the square of the covariant derivative. This is sensible, because these terms are minimized when the system is in its ideal configuration. In the first two systems, when the covariant derivative is squared, the cross term is a total divergence. When the free energy density is integrated over the whole sample, the total divergence can be integrated exactly and becomes a surface term. This surface term is evaluated at the boundaries of the sample, where it is insignificant in the thermodynamic limit. However, this term also contributes around all defects in the sample, and thereby effectively renormalizes the defect energy. If the net defect energy is sufficiently negative, the sample will have a finite density of defects. These defects are the gaps in the ripple phase, the boojums in HOBACPC, and the networks of disclination lines in the blue phases and metallic glasses.

$* * *$

This work was supported by National Science Foundation Grant No. DMR-8451921.

(5) The derivative has one index for the dimensions of space and one for the components of the order parameter.

${ }^{6}$ ) Given the form of the order parameter and the symmetries of the system, it is straightforward to write down the most general allowed covariant derivative. For the chiral films, the most general covariant derivative is $\partial_{i} \phi-q R_{i j} c_{j}$, where $R_{i j}$ is a rotation matrix. Using something other than $R_{i j}=\delta_{i j}$ simply shifts the frustration from the bend to the splay term in the free energy, without changing the basic physics. For the blue phase the covariant derivative in table I is the most general, given spherical symmetry.

\section{REFERENCES}

[1] Carlson J. M. and Sethna J. P., to be published in Phys. Rev. A.

[2] Langer S. A. and Setinna J. P., Phys. Rev. A, 34 (1986) 5035.

[3] Nelson D. R., Phys. Rev. B, 28 (1983) 5515.

[4] Sethna J. P., Phys. Rev. B, 31 (1985) 6278.

[5] See, for example, SACKMAN E., RUPPLE D. and GebhaRdT C., in Liquid Crystals of One and Two Dimensional Order, edited by W. HELFRICH and G. HEPPKE (Springer-Verlag, New York, N.Y.) 1980 , p. 309.

[6] ZASADZINSKI J. and SchNeider M., to be published.

[7] Janiak M. J., SMall D. M. and ShIPLEY G. G., Biochem., 15 (1976) 4575.

[8] Young C. Y., Pindak R., Clark N. A. and Meyer R. B., Phys. Rev. Lett., 40 (1978) 737.

[9] Rosenblatt C., Meyer R. B., Pindak R. and Clark N. A., Phys. Rev. A, 21 (1980) 140.

[10] Pindak R., Young C. Y., Meyer R. B. and Clark N. A., Phys. Rev. Lett., 45 (1980) 1193.

[11] van Winkle D. H. and Clark N. A., Phys. Rev. Lett., 53 (1984) 1157. 